\title{
Teoría de la modernidad y forma estética en la distinción de Walter Benjamin entre tragedia y Trauerspiel
}

\author{
Theory of modernity and aesthetic form in Walter Benjamin's \\ distinction between tragedy and Trauerspiel
}

\author{
Mariela Silvana Vargas \\ lublinskaya@yahoo.com.ar \\ (Universidad Nacional de Salta, Salta, Argentina)
}

\begin{abstract}
Resumen: El objetivo de este trabajo es mostrar que la distinción trazada por Walter Benjamin entre tragedia y Trauerspiel no contiene solamente una interpretación de las particularidades propias de cada forma dramática, sino que la distinción misma constituye uno de los fundamentos de su teoría de la modernidad. A través de una comparación de la teoría benjaminiana de la tragedia con la reflexión romántica y de principios del siglo XX sobre la tragedia se analizará la manera en que los conceptos de modernidad y tragedia están enlazados sistemáticamente en la obra de Benjamin, tanto desde el punto de vista genealógico, como desde el punto de vista problemático.
\end{abstract}

Palabras clave: modernidad; secularización; Walter Benjamin; Trauerspiel; tragedia.
Abstract: The aim of this work is to show that Walter Benjamin's distinction between tragedy and Trauerspiel contains not only an interpretation of the particularities of each dramatic form, but that the distinction itself constitutes one the fundaments of his theory of modernity. Through a comparison between Benjamin's theory of tragedy, the reflection on tragedy from romantics and from the beginning of 20th century, it will be analyzed how the concepts of modernity and tragedy are systematically intertwined in Benjamin's work, both from a genealogical and from a systematic point of view.

Keywords: modernity; secularization; Walter Benjamin; Trauerspiel; tragedy.

DOI: http://dx.doi.org/10.11606/issn.2318-9800.v23i1p95-106

\section{Introducción}

El núcleo problemático que articula el vínculo entre tragedia y modernidad está presente en los más disímiles diagnósticos políticos, estéticos y culturales de la modernidad, los que contienen siempre alguna reflexión sobre la naturaleza de la tragedia y lo trágico. Tanto los críticos como los defensores de la modernidad coinciden en una tesis que, en mayor o menor grado, ha permanecido implícita en las discusiones sobre el núcleo de lo moderno: la época moderna es aquella que viene después de la época de la tragedia o, en otras palabras, la modernidad es la época en 
la que la tragedia ya no es posible. Los conceptos de modernidad y tragedia parecen estar entrelazados sistemáticamente tanto desde el punto de vista genealógico, como desde el punto de vista problemático. El vínculo intrínseco entre tragedia y modernidad se torna visible en el plano histórico si se considera que la tragedia ha sido objeto privilegiado de una tradición que se remonta a dos siglos y en la que se inscriben las obras de Schelling, Hegel, Nietzsche, Simmel, Lukács, Benjamin, Gehlen, Foucault, entre otros. Desde mediados del siglo XVIII hasta principios del siglo XX la reflexión sobre los rasgos propios de la modernidad fue inseparable de la pregunta por la estructura de la tragedia.

Siguiendo la reconstrucción que lleva a cabo Christoph Menke (2001) del vínculo entre modernidad y tragedia es posible distinguir dos paradigmas en la teorización sobre la tragedia: un paradigma "romántico", que surge en el siglo XIX y traza un arco que va desde la Ilustración y el idealismo hasta Nietzsche; y un paradigma "mítico", que se despliega en el primer tercio del siglo XX e incluye a la naciente sociología con Max Weber, la obra de Carl Schmitt y algunas contribuciones de la Teoría Crítica en formación, con repercusiones hasta la década del cincuenta. Mientras que en el paradigma romántico la figura de la tragedia marca el inicio de la modernidad, en el paradigma mítico, es la figura de su fin. De acuerdo a éste último la tragedia encarna una instancia de desmoronamiento de la modernidad, la tragedia deviene en esta perspectiva la formación "a la que la modernidad retrocede" (Menke, 2001, p. 207) y constituye una suerte de producto secundario del fracaso y crisis de la modernidad. Por el contrario, para el modelo romántico la tragedia señala un punto de progreso; ella representa "el mundo del que proviene la modernidad a través de su superación" (idem, íbidem). Mientras que el modelo mítico habría tenido una vida relativamente corta, el modelo romántico y su tesis de la modernidad como época post-trágica constituiría para Menke el fundamento no declarado de los debates actuales sobre la modernidad y la posmodernidad.

El libro de Walter Benjamin sobre el origen del Trauerspiel (1926) se inscribe en una zona intermedia, en el umbral entre el conjunto de teorías sobre la tragedia provenientes de la tradición romántica y la estela de reflexiones surgidas en el paradigma mítico, aunque, como intentará mostrarse, no responde estrictamente a ninguno de los dos. Si bien para Benjamin allí donde hay modernidad no hay tragedia, no es posible afirmar que el fracaso moderno desemboque en la tragedia, ni adscribirle a la modernidad un fundamento o incluso alguna continuidad con elementos trágicos. En este sentido, el esfuerzo analítico desarrollado en la distinción benjaminiana entre tragedia y Trauerspiel apunta no sólo a la identificación de las particularidades propias de cada forma dramática, sino también a la delimitación histórica, teológica y política de una configuración específicamente moderna. 


\section{La distinción entre Trauerspiel y tragedia}

En la década previa a la redacción de su libro Origen del Trauerspiel alemán Benjamin dedicó algunos breves ensayos a algunos aspectos del mundo griego, ${ }^{1}$ entre los que se encuentra un texto titulado Trauerspiel y tragedia (II, pp. 137141), redactado en el verano de 1916. Si bien estas exploraciones juveniles guardan algunas diferencias con las afirmaciones vertidas más tarde en el libro sobre el Trauerspiel (I, pp. 259-307), en ambos textos están presentes dos rasgos distintivos del análisis benjaminiano de los productos literarios, estéticos y culturales: la incorporación de un punto de vista proporcionado por una consideración filosófica de la historia [geschichtsphilosophisch] y no sólo estética y una interpretación que combina el estudio empírico de los fenómenos con la discusión de las interpretaciones contemporáneas sobre éstos. ${ }^{2}$ Así, en una carta a Hans Reichenbach escrita en 1916, durante su época de estudiante en Múnich, Benjamin refiere haberse ocupado en aquel tiempo del "significado sistemático de la filosofía de la historia y en conexión con ello de la mitología" (GB 1, p. 349) y haber iniciado con ello un campo de "trabajo filosófico fructífero" (idem, íbidem). Ambos temas se constituirán en categorías fundamentales, que atraviesan la totalidad de la obra benjaminiana y que son determinantes para la comprensión de la perspectiva desde la que desarrolla la distinción entre Trauerspiel y tragedia.

Ya en el ensayo juvenil Benjamin afirma que una auténtica comprensión de lo trágico no debe "partir del arte, sino de la historia" (II, p. 137) y señala, más específicamente, que la diferencia entre Trauerspiel y tragedia reside en la posición que adopta cada género "ante el tiempo histórico" (II, p. 138). Para explicar esta tesis Benjamin desarrolla una distinción entre los conceptos de tiempo histórico, tiempo trágico y tiempo mesiánico: mientras que el tiempo de la historia es "infinito en cada dirección y está sin consumar en cada instante” (idem, íbidem), el tiempo mesiánico es un "tiempo divinamente consumado" (erfüllt) (idem, íbidem). Existe sin embargo, un tiempo individualmente consumado, el tiempo trágico. La tragedia

1 Cf. Benjamin, W. La felicidad del hombre antiguo; Sócrates; Trauerspiel y tragedia; El significado del lenguaje en el Trauerspiel y la tragedia (Benjamin, 2006, pp. 130-133; 133-136; 137-141; 141 144). Las obras de Benjamin se citarán con el número de tomo en cifras romanas y la página en números arábigos.

2 Así, por ejemplo, el escrito juvenil discutía implícitamente con la conferencia ofrecida por Martin Heidegger en la Universidad de Friburgo en el verano de 1915 sobre El concepto del tiempo en la ciencia histórica (Heidegger, 1978. pp. 413-433) y con El problema del tiempo histórico de Georg Simmel (1916), mientras que el libro sobre el Trauerspiel recogía y discutía las teorías más importantes de su tiempo sobre la tragedia - El nacimiento de la tragedia de Friedrich Nietzsche; Estética de lo trágico de Johannes Volkelt; Metafísica de lo trágico de Leopold Ziegler; Metafísica de la tragedia de Georgy Lukács y la Estrella de la Redención de Franz Rosenzweig - y las reconstrucciones más influyentes sobre el rol de la Antigüedad en la constitución del mundo moderno, en particular los textos de Jakob Burckhardt Griechische Kulturgeschichte y Karl Borinski Die Antike in Poetik und Kunsttheorie vom Ausgang des klassischen Altertums bis auf Goethe und Wilhelm von Humboldt. 
es así definida como el espacio donde se produce el paso del tiempo histórico al tiempo trágico. Dado que Benjamin no especifica si dicha transformación es algo exclusivo de la obra de arte, la ubicación de la tragedia en el plano estético o en el de la historia queda sin determinar.

Este tiempo consumado, el tiempo del héroe, no conoce la contingencia e indeterminación propias del tiempo histórico, pero no se halla por ello tampoco situado estrictamente en el plano del tiempo mesiánico, empíricamente indeterminado por completo. El tiempo trágico se entremezcla parcialmente con el tiempo histórico y el tiempo mesiánico; es un tiempo sobredeterminado, y es este aspecto precisamente el que lo hace trágico, pues "en el tiempo consumado no hay nadie que sea capaz de vivir" (II, p. 138). Es por este motivo que Benjamin afirma que la muerte del héroe es, irónicamente, una muerte por inmortalidad. Tal es el origen de la ironía trágica (II, p. 138). Mientras que en la tragedia la muerte del héroe es una manifestación de los dioses en el mundo humano y natural y de la compleción de y en el tiempo, en el Trauerspiel la muerte no está ligada a la consumación, pues ésta ha ocurrido ya en el nacimiento de Cristo y en el siempre todavía venidero Juicio Final.

Hacia 1924, cuando inicia la redacción del Origen del Trauerspiel alemán, Benjamin retoma las ideas centrales sobre la diferencia entre Trauerspiel y la tragedia de su primer ensayo y las desarrolla en direcciones novedosas, adoptando, además, un nuevo punto de partida: la tesis de la imposibilidad de la modernidad para producir tragedias, esto es, la tesis del carácter históricamente situado e irrepetible de la tragedia. Esta tesis, que no estaba presente en el primer ensayo, y que marca la principal diferencia entre ambos trabajos, condujo a Benjamin a una comprensión de la modernidad que marcaría también el proyecto del Libro de los Pasajes, consistente en la escritura de una Ur-Geschichte de la modernidad. En su estudio sobre el barroco Benjamin concluye que el siglo posterior a la reforma protestante es de capital importancia para comprender las transformaciones que definen a la modernidad y que autores como Reinhart Koselleck sitúan mucho más tarde, en el Sattelzeit, el último tercio del siglo XVIII (Koselleck, 2004). En su rastreo minucioso de la imaginación barroca Benjamin parece seguir la idea hegeliana de que las grandes revoluciones, las que impresionan los ojos de todos, son precedidas de revoluciones silenciosas que "no son observables para todos los contemporáneos y que son tan difíciles de presentar como de comprender" (Hegel, 1907, p. 220). El libro sobre le Trauerspiel puede leerse así como el trabajo de representación (Darstellung) de las transformaciones fundamentales, de los cambios espirituales y teológicos que están a la base de las revoluciones modernas.

En el libro sobre el drama barroco y en el marco de su programática incorporación de la filosofía de la historia para el estudio de la diferencia entre Trauerspiel y tragedia, Benjamin presta especial atención a la situación teológica 
de cada época y al vínculo que el hombre establece con la divinidad y lo absoluto en cada una de estas formas estéticas. El contenido de la tragedia consiste para Benjamin en la "actualización de un pasado inmemorial" (I, p. 265), cuyo objeto es el mito y cuyo desarrollo se produce bajo la forma del "enfrentamiento con Dios y el destino" (idem, íbidem). El punto álgido de ese enfrentamiento está dado por la muerte del héroe, que constituye el sacrificio de una humanidad que, de esa manera, se libera de la coacción de las fuerzas míticas. Es precisamente este duelo decisivo del mundo griego con el orden demónico [dämonische Weltordnung], con la inmanencia implacable del destino, lo que le otorga a la poesía trágica su signatura en términos de filosofía de la historia [geschichtsphilosophische Signatur] (I, p. 318).

Mientras que el príncipe muere "en nombre de la humanidad histórica" y su caída se produce "como un veredicto de cuyo fallo el súbdito se siente copartícipe" (I, p. 276), el héroe trágico muere en nombre de una comunidad venidera. La poesía trágica descansa en la idea de sacrificio, pero este sacrificio posee la particularidad de que su objeto es el héroe mismo y por ello constituye al mismo tiempo un principio y un final. "Un final porque es un sacrificio expiatorio debido a los dioses, guardianes de la ley antigua; un principio porque se trata de una acción sustitutiva en la que se anuncian nuevos contenidos de la vida del pueblo" (I, p. 315). Benjamin señala que estos nuevos contenidos, a diferencia de las antiguas sujeciones míticas, no emanan de un mandato superior, sino que nacen de la vida del héroe mismo, y por ello mismo terminan aniquilándolo, ya que, por ser "desproporcionados a la voluntad individual, benefician solamente a la vida de la comunidad popular aún por nacer" (I, pp. 315s). En este punto se comprende el doble significado que posee la muerte trágica: debilita la ley antigua de los dioses olímpicos y ofrenda el héroe a un dios desconocido y venidero.

A pesar de las paradojas que Benjamin señala en la tragedia, tales como el hecho de que "en el sacrificio, que, cumpliendo con la vieja ley, funda una nueva; en la muerte, que es expiación, pero que sólo consigue arrebatar el yo; en la conclusión, que decreta la victoria para el hombre y también para el dios", constata también que "la ambigüedad, señal inconfundible de lo demoníaco, se halla en estado de extinción” (I, p. 318). La tragedia, que parecía consistir en un juicio al héroe, se transforma en un proceso a los olímpicos en el que, contra la voluntad de los dioses, se proclama “el honor del semidiós” (I, p. 319). En la tragedia se consigue quebrar el destino mítico, pero "no porque el infinito encadenamiento pagano de culpa y expiación fuera sustituido por la pureza del hombre absuelto y reconciliado con el dios puro, sino porque en la tragedia el hombre pagano advierte que es mejor que sus dioses" (idem, íbidem). Benjamin subraya la idea de que en la tragedia no se trata de restaurar el 'orden ético del mundo', y que el gran logro del hombre pagano es que "levantarse en medio de la inestabilidad de aquel mundo atormentado" (idem, 
íbidem).

El énfasis en la autoafirmación humana que tiene lugar en la tragedia, aún cuando se limite al héroe y como profecía dirigida a su comunidad, es descrita como quiebre del orden demónico, y así, como acto secularizador. En La legitimación de la Edad Moderna Hans Blumenberg (2008) define la secularización como acto de autoafirmación de lo humano ante el ‘absolutismo teológico’ propio de las postrimerías de la Edad Media, esto es, contra el poder total de un dios inasible y contra una naturaleza, cuyo orden y estructura no puede ser ya garantizado por aquél, por lo que se le presenta al hombre como indisponible e incontrolable. ${ }^{3}$ Pero mientras que para Blumenberg los conceptos de autoafirmación y autoconstitución son imposibles de formular en la Antigüedad, Benjamin sitúa justamente aquel acto en el corazón del mundo antiguo. El héroe trágico se autoafirma contra los dioses y el destino, es decir, lleva a cabo un logrado acto de secularización. ${ }^{4}$

Por el contrario, el contenido del Trauerspiel no es ya el mito, sino la "vida histórica", encarnada en el Príncipe, pero desligada de un vínculo unívoco y seguro con el más allá. Para Benjamin el Dios del barroco representa apenas un polo, un elemento más de la tensión, definitoria de la época, entre mundo y trascendencia. El enfrentamiento del príncipe con un mandato absoluto, expresado en el ideal de un supremo poder ejecutivo, una dictadura cuya "utopía" consistía en "sustituir el errático acontecer histórico" (I, p. 277) por las férreas leyes naturales, lo conducen a una situación de pérdida de la soberanía sobre sí mismo y sobre los otros, marcada por la incapacidad de decidir. La muerte del soberano no produce un héroe, sino un mártir, que, en la medida en que sucumbe a sus propios impulsos y pasiones, expone la naturaleza creatural del hombre, y por ello mismo, necesitada de redención: "víctima de la desproporción de la ilimitada dignidad jerárquica con que Dios lo inviste, cae en el estado correspondiente a su pobre esencia humana” (I, p. 274). En el drama barroco la muerte del príncipe no posee una función trágica, ni prepara un camino secularizador de liberación de un orden demónico. La temporalidad dramática es concebida como presente intramundano, en el que no hay lugar para un instante trágico, ni para el tiempo mesiánico. En el drama barroco la muerte no es manifestación de un orden superior que gobierna sobre la vida y la muerte, y no constituye por lo tanto un fin, sino el inicio de una repetición y continuación espectral de la muerte, al tiempo que la redención permanece como una promesa, una figura situada en el más allá.

3 Para una discusión sobre el carácter ilegítimo de la categoría de secularización en Blumenberg ver la entrada "Säkularisierung" del Glosario sobre Blumenberg editado por Weidner y Buch (2014, pp. 547ss).

4 Con esta interpretación de la tragedia griega como acto secularizador Benjamin se acerca a la postura presentada por Hermann Cohen en su Ästhetik des reinen Gefühls (Cfr. Cohen, 1982, pp. 80ss). 


\section{Estética y Filosofía de la Historia}

En la teoría benjaminiana de la tragedia se tornan visibles los blancos de su polémica contra las teorías dominantes en la época. En primer lugar, la postura de Benjamin presenta diferencias importantes con el 'paradigma romántico' de la tragedia, basado en la contraposición entre tragedia y reflexividad. Según éste, la modernidad es un logro de la autorreflexión del héroe, que desemboca en la superación de la tragedia por la comedia. Esta afirmación, sin embargo, como señala Menke, encierra una tesis más fuerte: "el «y» de esta doble afirmación -logro de la reflexividad y superación de la tragedia - tiene que leerse como un doble «mediante», de modo que la modernidad es logro de la reflexividad mediante la superación de la tragedia y superación de la tragedia mediante el logro de la reflexividad" (Menke, 2001, p. 207). Benjamin, por el contrario, no establece una conexión necesaria entre modernidad y reflexión y desplaza el acento en la interpretación del momento en el que el héroe trágico toma consciencia del conflicto en el que se halla hacia el enfrentamiento exitoso del héroe con las fuerzas míticas, lo que conduce no sólo a la consciencia del héroe de su superioridad frente a los dioses, sino fundamentalmente a una experiencia de la temporalidad plena insostenible por una vida individual. La muerte del héroe no obra el paso hacia una instancia autorreflexiva en la que se diluyen los conflictos ético-políticos, sino que representa un instante de liberación del mito y de plenitud. El momento de consumación o realización plena en el tiempo de un individuo, esto es, la existencia de un tiempo trágico, constituye el elemento singular y propio de la tragedia y de la antigüedad.

Benjamin rechaza también la idea de que la tragedia se basa en un "sistema de sentimientos universales" (I, p. 308). Tal idea equivaldría a sostener que lo trágico puede darse "en ciertas constelaciones fácticas que ocurren en la vida" (idem, íbidem), es decir, que en los conflictos trágicos se expresa un "contenido humano universal" (I, p. 309), pasible de ser actualizado en diferentes momentos de la historia. Tal tesis sustentaba el intento de desarrollar una "filosofía de la tragedia" desligada de la consideración de los “contenidos históricos objetivos” (I, p. 308), que serían específicamente griegos. La escena moderna no ofrece tragedias como las de los griegos ${ }^{5}$ pues la tragedia no puede ser repetida ni revivida. ${ }^{6}$ Con esta afirmación se pone de manifiesto la distancia que separa a Benjamin de los representantes del 'paradigma mítico' de la tragedia y su tesis de una recaída de la modernidad en lo

\footnotetext{
5 Cf. "El sello distintivo de la tragedia no está constituido por un simple «conflicto de niveles» entre el héroe y el mundo circundante, como Scheler afirma en su estudio Sobre el fenómeno de lo trágico, sino por el carácter singularmente griego que adoptan tales conflictos" (I, p. 315).

6 Peter Fenves señala que para Benjamin, "La tragedia es una forma dramática única y que hace época, destinada a no ser repetida y mucho menos revivida en términos de 'lo trágico'" (Fenves, 2001, p. 229).
} 
trágico. No hay sin embargo en Benjamin una teoría de la modernidad formulada de manera enfática y positiva, sino que los elementos constitutivos de la modernidad se tornan visibles ex negativo, en contraste con la caracterización que lleva a cabo de la tragedia.

Cuando Benjamin le niega a la modernidad aquellas condiciones que harían posible la tragedia, está negando también en el fondo el éxito del proyecto secularizador, con el que la modernidad se ha caracterizado frecuentemente a sí misma. Tras el desmoronamiento de las esperanzas de redención del monoteísmo la modernidad se parece, para decirlo con Max Weber, al "viejo mundo, todavía no desencantado con sus dioses y demonios": "los viejos dioses, encantados y por ello en forma de potencias impersonales, se levantan de sus tumbas, aspiran a dominar sobre nuestras vidas y comienzan de nuevo su lucha eterna" (Weber, 2010, p. 470). La estructura religiosa e incluso mítica de la modernidad capitalista ya había sido objeto de las reflexiones de un fragmento redactado en 1921 y titulado Capitalismo como Religión y continuó ocupando un lugar central en el Libro de los Pasajes, cuyas primeras anotaciones datan de 1927. Tanto el Libro de los Pasajes como el libro sobre el Trauerspiel establecen una correlación entre los procesos de secularización en el barroco y en la modernidad, en la medida en que, de acuerdo con Benjamin, ambos representan intentos fallidos de comprender y actuar sobre la historia (Cfr. Benjamin, 2005, pp. 475; 284; 219).

El libro sobre el Trauerspiel revoca también el proyecto de sujeto que había surgido al cobijo del proceso de secularización, caracterizado por un giro hacia lo terrenal. Pero mientras que desde el punto de vista de la tragedia sentido y ser son idénticos y ella se convierte en el símbolo del destino y la finitud del hombre, en el Trauerspiel el destino del hombre se encuentra confrontado con la crisis de la promesa cristiana de salvación, con la "desaparición de toda escatología” (I, p. 285) y su deriva melancólica. La tendencial disminución de reflexiones sobre la trascendencia no era producto de la ausencia de preocupaciones teológicas, sino expresión del quiebre de la unidad de sentido y su traslado al mundo de la apariencia. Para el sujeto (post-trágico) barroco todos los intentos de una fundamentación certera del sentido, a la vez que su misma posición como sustancia subyacente, se revelan como vanos, y con ello la posibilidad de afirmarse ante un Dios y un mundo insondables se encuentra también puesta en cuestión. El sujeto benjaminiano moderno no es un sujeto reflexivo ni soberano.

En el barroco Benjamin reconoce ya la inflación de signos cuyo sentido había dejado de ser unívoco, característica de la Modernidad. De ahí la afinidad de la alegoría con la melancolía y su nostalgia por un mundo regido por lo divino. La alegoría y el soberano son las dos figuras que condensan la reacción a la crisis barroca del proceso de secularización. En la figura del alegorista Benjamin cuestiona al sujeto burgués 
garante de un orden racional del mundo, y en el énfasis en la labilidad e impotencia del soberano muestra los límites de la elaboración político-filosófica con la que en el siglo XX se intentó dar respuesta a la puesta en cuestión del sujeto: la idea de Carl Schmitt de un soberano absoluto y los proyectos de restauración conservadora de algunos críticos de la modernidad. El Trauerspiel no conoce héroe alguno, sino sólo constelaciones (I, p. 310), por ello la figura central es la del alegorista. Sin embargo, el intento de obtener de las cosas algún sentido que remita a algo más allá de sí mismas a través de la reflexión está condenado a fracasar, pues el alegorista encuentra siempre solo aquel sentido que él mismo le confirió a las cosas. El alegorista pone en evidencia la carencia de fundamento de aquel acto de autoafirmación humana, que tuvo lugar en parte porque la divinidad se había vuelto inescrutable. El hiato entre los signos y el significado se había abierto de manera definitiva e irrevocable: "cada persona, cada cosa, cada relación puede significar cualquier otra cosa" (I, p. 350). ${ }^{7}$ Precisamente allí donde la autonomía del sujeto se convierte en "impotencia" (I, p. 275), se encuentra la línea que conecta al soberano del Trauerspiel con el alegorista, pues el soberano es aquel que, en la concepción schmittiana, dispone del poder absoluto de la determinación del significado en la decisión sobre el Estado de Excepción. Benjamin, sin embargo, introduce una distinción entre el poder del gobernante [Herrschermacht] y el poder de gobernar [Herschervermögen], que pone al descubierto la desproporción entre la "ilimitada dignidad jerárquica” con que Dios inviste al príncipe y su “pobre esencia humana” (I, p. 274), lo que conduce a su caída. ${ }^{8}$ La distancia entre poder del gobernante y el poder de gobernar es complementaria de la distancia entre signo y significado. La imposibilidad de clausurar la brecha entre esos términos, produce una "hipérbole teológica" (I, p. 270), es decir, una elipse con un extremo que se extiende indefinidamente, mientras el otro permanece en el espacio inmanente del mundo finito.

A diferencia de la forma simbólica, cerrada y unitaria de la tragedia, que conduce al destino inexorable del héroe, la forma abierta y alegórica del Trauerspiel señala más allá de sí misma. En el ensayo juvenil sobre la distinción entre tragedia y Trauerspiel, Benjamin pone de relieve el hecho de que en éste la muerte no se basa

\footnotetext{
7 Benjamin discute aquí con Friedrich Schlegel, quien en Diálogo sobre la poesía, sitúa el arte bajo el signo de la alegoría: "toda belleza es alegoría. Lo más elevado [das Höchste], puesto que es inexpresable [unaussprechlich], sólo puede ser pronunciado alegóricamente" (Schlegel, 2005, p. 71). También lo más elevado (das Höchste), consistente en la superación del hiato entre el mundo sensible y el mundo moral es concebido dentro del horizonte de la belleza y por lo tanto, de modo alegórico. El paso de un dominio a otro ocurre en un giro o vuelco (Umschlagen). Schlegel no explica cómo la alegoría, que se mueve en el mundo de las distinciones y delimitaciones, y con ellas, de la referencia nominal, puede producir una referencia o nominación sustantiva. Benjamin por el contrario, niega tanto el carácter progresivo que el romanticismo le adjudicaba a la alegoría como la capacidad de producir un significado sustancial, que la acercaría al símbolo. La alegoría sólo puede comprenderse para Benjamin como alegoría de la alegoría.

8 Por otra parte, si el soberano acabara efectivamente con el estado de excepción, se diluiría con ello la base de su soberanía.
} 
en esa determinación extrema que el tiempo individual le confiere al acontecer y no constituye ella misma un final: "sin certeza en la vida superior y sin ironía, dicha muerte es metábasis de toda vida eis állo génos" (II, p. 140). Esta expresión aristotélica, que significa "cambio de género", alude a una figura o estrategia argumentativa en la que el desarrollo de un objeto conduce, según su ley propia, a una configuración 'más allá de la serie' y efectúa así un salto a otro género, esfera o categoría. ${ }^{9}$ Esta idea es continuada en Origen del Trauerspiel alemán, en el que Benjamin se adentra en el estudio de un mecanismo alegórico, por el cual la separación absoluta que supone la alegoría entre presencia y representación, puede ser revertida y que Benjamin designa como "vuelco" o "giro" [Umschlagen] (I, p. 455; p. 456). En este giro la forma de referencia o remisión alegórica pasa de la pura inmanencia carente de fondo a la presencia de un sentido infinito.

En este punto podría parecer que la teoría benjaminiana sobre el drama barroco posee un elemento común con otra teoría derivada del paradigma romántico sobre la tragedia, que postula la autonomización postrágica del juego estético a través del que se efectúa la presentación misma de los conflictos como fenómeno distintivo de la modernidad. La autorreflexión de la exposición convierte a ésta en juego estético, en el que se diluye la experiencia ética, dando lugar al surgimiento de la comedia (Cfr. Menke, 2001). Complementaria a la teoría del sujeto moderno como autoconsciencia racional y autónoma, la teoría del juego estético postula que lo que deviene autónomo es el juego mismo. En la teoría alegórica de Benjamin, sin embargo, no se produce una autonomización completa del juego, pues el juego alegórico no pierde su condición mundana de "juego del mundo", por lo que la obra de arte alegórica, en tanto "obra que está haciéndose eternamente a sí misma" (Schlegel, 2005, p. 80), permanece como el reverso, como lo otro de la presencia de un sentido infinito y no puede constituir así fundamento firme alguno. El Trauerspiel presenta una forma inacabada, incompleta, por lo que no puede constituir una forma autonomizada. La forma de la referencia alegórica está confinada a la inmanencia y eso significa también que el acceso a su propio carácter construido es siempre alegórico, como alegoría de la alegoría.

El modo en que la palabra Trauerspiel, es tomada en toda su literalidad y descompuesta en los conceptos que la componen: Trauer - duelo- y Spiel, juego, obra resume el tratamiento benjaminiano de este género dramático. Entendido como teatro luctuoso el Trauerspiel es definido como obra para melancólicos y dedicada a satisfacer la melancolía (II, p. 298). Pero la melancolía no es tanto la vivencia de un sujeto, como una estructura del mundo (Ferber, 2013). No hay primacía del sujeto sobre el objeto artístico y éste tampoco es el producto de una subjetividad que

9 "El Trauerspiel es en esto comparable matemáticamente a una de las ramas de la hipérbola, cuya otra rama se encuentra en lo infinito" (II, p. 140). 
se exprese a sí misma. El Trauerspiel se distancia del concepto de representación encarnada; su eje es la exposición de imágenes, destinadas a ser expuestas. Se trata de un teatro cuyo sentido no está dado por la estructura dramática o los personajes, sino por la teatralidad misma, a la vez que es un teatro dirigido a un público cuya relación con la teatralidad es a su vez, teatral (Menke, 2010). El libro sobre el Trauerspiel puede considerarse también producto del rechazo benjaminiano a la entronización de la experiencia subjetiva como categoría estética (II, p. 276) y una muestra del modo en que es posible reemplazarla por la relación objetiva entre historia y estética a la hora de interpretar obras de arte.

Finalmente, puede verse en el libro sobre Trauerspiel una reacción a la crisis de la representación política en la República de Weimar. La concepción que Benjamin presenta de la modernidad en esta obra se halla en radical oposición a una concepción continuista o sustancial de la historia, y su noción de la subjetividad, encarnada en la figura inestable el soberano, aparece dislocada y vulnerable a los peligros y excesos de la embriaguez [Rausch] y las emociones violentas. La modernidad benjaminiana no es la modernidad de un sujeto autorreflexivo inmerso en una única y continua historia universal, tal como lo será en Habermas o Menke, quienes enfatizan la dimensión política de la experiencia estética y hasta su potencial democrático. En su intento de salvar el Trauerspiel como 'Idea', como fragmento, como forma que no acaba de formarse y que permanece inconclusa (I, p. 459), Benjamin muestra de qué manera la consumación de la vida, su representación en una forma, constituía el auténtico problema de la modernidad, el del misterio sin teología.

\section{Referencias}

Benjamin, W. (1997). Gesammelte Briefe. Fráncfort del Meno: Suhrkamp. . (2005). Libro de los Pasajes. Madrid: Akal. - (2006). Obras. Libro I. Vol 1. Madrid: Abada. . (2007). Obras Libro II. Vol. 1. Madrid: Abada.

Blumenberg, H. (2008). La Legitimación de la Edad Moderna. Valencia: Pre-Textos.

Caygill H. (1994). Walter Benjamin's Philosophy. Destruction and Experience. Londres / Nueva York: Routledge.

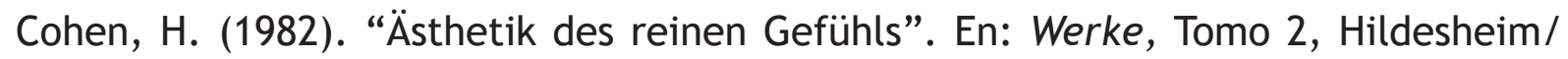
Nueva York: Georg Olms Verlag.

Fenves, P. (2001). Arresting Language. From Leibniz to Benjamin. Stanford: Stanford University Press.

Ferber, I. (2013). Philosophy and Melancholy. Stanford: Stanford University Press.

Hegel, W. (1907). “Diferencia entre la imaginación religiosa griega y la religión positiva cristiana”. En: Nohl, H. (ed.). Hegels theologische Frühschriften. Tubinga: J. C. B. Mohr. 
Heidegger, M. (1978). Gesamtausgabe Band I, Frühe Schriften. Fráncfort del Meno: Vittorio Klostermann, pp. 413-433.

Koselleck, R. (2004). historia/Historia. Madrid: Trotta.

Menke, B. (2010). Das Trauerspiel-Buch. Der Souverän - das Trauerspiel Konstellationen - Ruinen. Bielefeld: Transkript.

Menke, C. (2001). Conflicto ético y juego estético. Acerca del lugar histórico filosófico de la tragedia en Hegel y Nietzsche. Traducción de Gerard Vilar. En: Enrahonar, 32/33, pp. 203-222.

Schlegel, F. (2005). Diálogo sobre la poesía. Traducción de Laura Carugatti. Buenos Aires: Biblos.

Simmel, G. (1916). Das Problem der historischen Zeit. Berlín: Reuther\&Reichard.

Weber, M. (2010). “La ciencia como vocación”. En: Obras Selectas. Buenos Aires: Distal.

Weidner, D. (2014). “Säkularisierung”. En: Weidner, D.; Buch, R. (eds.). Blumenberg lesen: Ein Glossar. Berlín: Suhrkamp. 